\title{
Проблемы оптимизации
} энергопотребления домохозяйств

\section{в задачах повышения энергоэффективности жилищного сектора}

Целью работы является исследование проблемы оптимизации энергопотребления и практического применения методов повышения энергоэффективности в жилищном секторе. Оптимизация управления энергоэффективностью позволяет уменьшать расходование энергоресурсов при выполнении различных работ, отопление зданий и т.д. Создание методов оптимизации позволит в короткие сроки снизить платежи за коммунальные услуги, а в целом для отрасли, будет способствовать уменьшению потребления различных ресурсов и улучшению экологического состояния региона. В отличии от других подходов, акцент в данной работе ставится на удобство и простоту, необходимую для использования этой методики населением в домашних хозяйствах.

В предложенном комплексном подходе используются методы теории вероятностей, линейного программирования, модели теплообмена. Проведенное исследование подтверждает эффективность полученного решения и может служить основой для создания учебно-исследовательских стендов.

Статья состоит из двух частей: в первой части выполнен анализ ведуших работ в этой тематике и определены причины, затрудняющие массовое применение предлагаемых в этих работах решений. Далее предложена и обоснована постановка задачи и сформулирован ряд основных требований к математической модели энергопотребления, необходимых для того, чтобы сконструированную методику можно было применять для оптимизаиии энергопотребления в домашних хозяйствах. Во второй части на примерах конкретных бытовых электроприборов предлагается математическая модель их функционирования. При исследовании существуюших методов оптимизации энергопотребления в домохозяйствах были выявлены проблемы, заключаюшиеся в сложности применения этих методов на практике и получены рекомендации, позволяющие сформулировать основные принципы построения методики оптимизации, удобной для практического применения. Было показано, что при построении такой методики первичным является вопрос о данных, которые может предоставить пользователь. Был определен минимальный состав входных данных, по которым сконструированы необходимые алгоритмы оптимизации энергопотребления. Так же был предложен ряд алгоритмов определения некоторых входных показателей, которые легко использовать в домашних хозяйствах.

Таким образом, общий план исследований в данной работе заключается в следующем:

- провести группировку приборов по способу задания функциональных требований

- выяснить приемлемый для пользователя состав и вид входных данных;

- определить минимальный набор входных данных для формализации ограничения суммарной потребляемой мощности;

- сконструировать алгоритмы оптимизаиии, работающие с указанными выше входными данными.

Важнейшими результатами выполненной работы являются следуюшие:

- разработана методика прогнозирования графика максимальной суммарной мощности потребления.

- разработаны методики оптимизаиии энергопотребления для каждого из выделенных подмножеств бытовых приборов.

- выполнено моделирование полученных алгоритмов оптимизаиии, которое показало их работоспособность, эффективность и возможность их практического применения без какой-либо адаптации.

Таким образом, в статье предложено решение задачи оптимизации энергопотребления в жилищном секторе, ориентированное на практическое применение.

Ключевые слова: энергоэффективность, домохозяйство, профиль нагрузки

Georgiy G. Grebenuk, Sergey M. Nikishov, Andrey A. Krygin, Leonid A. Sereda V. A. Trapeznikov Institute of Control Sciences of RAS, Moscow, Russia

\section{Problems of optimizing the energy consumption of households in the tasks of improving the energy efficiency of the housing sector}

The aim of the work is to study the problem of optimizing energy consumption and practical application of methods for improving energy efficiency in the housing sector. Optimization of energy efficiency management allows to reduce the expenditure of energy resources in the performance of various works, heating of buildings, etc. The creation of optimization methods will make it possible to reduce payments for utilities in a short time, and in general for the industry, will help reduce the consumption of various resources and improve the ecological state of the region. Unlike other approaches, the emphasis in this paper is on the convenience and simplicity necessary for using this technique by the population in households.

The proposed integrated approach uses methods of probability theory, linear programming, heat exchange models. The conducted research confirms the effectiveness of the solution obtained and can serve as a basis for the creation of training and research stands. 
The article consists of two parts: the first part analyzes the leading works in this field and identifies the reasons that make it difficult to apply the solutions proposed in these papers. Further, the statement of the problem was proposed and justified, and a number of basic requirements to the mathematical model of energy consumption, necessary for the constructed technique to be used to optimize energy consumption in households, were formulated. In the second part, a mathematical model of their functioning is proposed using examples of specific household electrical appliances.

When researching existing methods for optimizing energy consumption in households, problems were identified that were difficult to apply these methods in practice and recommendations were obtained that allowed to formulate the basic principles of constructing an optimization technique that was convenient for practical application. It was shown that when constructing such a technique, the primary question is the data that the user can provide. The minimum composition of input data was determined, according to which the necessary algorithms for optimizing energy consumption were designed. A number of algorithms for determining some input indicators that are easy to use in households have also been proposed.

Thus, the general plan of research in this paper is as follows:
- carry out grouping of devices by the way of setting functional requirements

- determine the acceptable composition and type of input data for the user;

- define the minimum set of input data for formalizing the limitation of the total power consumption;

- design optimization algorithms that work with the input data specified above.

The most important results of the work performed are the following:

- the methodology for forecasting the graph of the maximum total power consumption has been developed.

- methods for optimizing energy consumption for each of the selected subsets of household appliances have been developed.

- the optimization algorithms obtained have been simulated, which showed their operability, efficiency and the possibility of their practical application without any adaptation.

Thus, the article proposes the solution of the problem of optimization of energy consumption in the housing sector, oriented to practical application.

Keywords: energy efficiency, household, load profile

\section{Введение}

На жилой сектор стран мира приходится значительный объем энергопотребления. Так по данным [Buildings Energy Data Book, Energy Efficiency and Renewable Energy: Building Technologies Program, U.S. Department of Energy, Washington, D.C., Mar. 2012] в 2010 жилой сектор США пришлось почти $40 \%$ от всей потребленной электрической энергии. Поэтому оптимизации энергопотребления в этом секторе экономики уделяется все большее внимание, а управление энергопотреблением, на взгляд авторов, должно стать предметом обучения на экономических отделениях университетов.

Предметом исследования данной работы являются вопросы, возникающие при реализации системы оптимизации энергопотребления в жилом секторе. Многочисленные публикации свидетельствуют об актуальности и важности этой задачи, одна из основных целей решения которой заключается в снижении неравномерности графика суточного потребления, включая снижении величины пикового потребления. Для ее достижения со стороны энергоснабжающих организаций применяется переменный тариф на электроэнергию в за- висимости от времени суток. Соответственно, со стороны потребителей имеется мотивация к снижению затрат путем изменения графика работы бытовых приборов таким образом, чтобы общая стоимость потребленной энергии была минимальной.

Подавляющее большинство научных работ на эту тему носят сугубо теоретический характер: обычно проводится формализация задачи, строится математическая модель, выводятся необходимые уравнения, неравенства, выбирается оптимизируемая функция. Потом выполняется компьютерное моделирование, с помощью которого находится оптимальный график и оценка экономической выгоды. Среди проанализированных ведущих работ в этой тематике [1-16] не обнаружилось ни одной, где бы говорилось о реальном применении разработанной авторами методики.

Цель данной работы заключается в выявлении причин в сложившейся ситуации и решении ряда вопросов, связанных с принципами построения методики оптимизации при практическом применении, таких как: формализация задачи оптимизации энергопотребления, достижение приемлемой вычислительной сложности ее решения и разработка алгорит- мов, необходимых при расчете оптимального графика.

Статья состоит из двух частей: в первой части предлагается и обосновывается постановка задачи и формулируется ряд основных требований к математической модели энергопотребления, во второй части на примерах конкретных бытовых электроприборов предлагается приемлемая для пользователя математическая модель их работы.

\section{1. Математическая модель оптимизации энергопотребления}

\section{1. Принципы построения математической модели}

Анализ работ [1-16] на предмет возможности их применения показал, что основная причина, по которой практическое применение изложенных в них методик затруднено, заключается в необходимости их серьезной адаптации к конкретной жизненной ситуации. Например, в [1] говорится об использовании полученных результатов при наличии инфраструктуры «умных сетей». Т.е. областью применения этих исследований является теоретическое обоснование предлагаемых методик, без учета ряда практических проблем. Эти проблемы в ходе анализа 
многочисленных работ были разделены на группы; условно назовем их: «проблемы входных данных» и «проблемы вычислительной сложности». Для дальнейшего их разбора, сформулируем в общих чертах задачу оптимизации и подход к ее решению.

Будем рассматривать следуюшую схему взаимодействия между потребителем и используемой им программой оптимизации энергопотребления:

1. Потребитель определяет группу оптимизируемых электроприборов;

2. Потребитель вводит в программу оптимизации все необходимые для расчета данные;

3. Выполняются необходимые расчеты, в результате которых пользователю предоставляется оптимальный набор графиков работы выбранных им электроприборов.

Назовем допустимым - график работы оптимизируемого прибора, удовлетворяющий пользователя. В дальнейшем будем называть пользовательские требования к работе прибора «функциональными требованиями». Ниже будет проведена формализация функциональных требований к электроприборам. Под оптимальным набором понимается сумма допустимых графиков всех оптимизируемых приборов, которая достигает оптимума по выбранному критерию или критериям и удовлетворяет некоторым ограничениям. Для РФ эти ограничения, как правило, сводятся к тому, что суммарная потребляемая мощность всех приборов (не только оптимизируемых) не должна превышать заданный максимальный порог, например, допустимая мощность энергоустановок в квартире в многоквартирном доме может ограничиваться значением 4,5 кВт. При превышении следуют штрафные санкции.

Общий подход к решению этой задачи заключается в сле- дующем: весь период, на котором проводится оптимизация, разбивается на равные интервалы (назовем их «интервалы оптимизации»). Величина интервала выбирается таким образом, чтобы в течение каждого интервала были постоянны все переменные модели: стоимость единицы энергии, суммарное потребление, режимы работы приборов и пр.; обычно длительность интервала оптимизации составляет 1-10 минут. Далее вводятся переменные, отражающие состояние прибора на каждом интервале оптимизации, и строится целевая функция и неравенства, описывающие функциональные требования к прибору. В результате задача сводится к одной из известных задач оптимизации, как правило, к задаче линейного целочисленного программирования.

Анализ этой схемы показал, что существует противоречие (которое выше и названо «проблема входных данных»): с одной стороны, чем более подробные данные по функциональным требованиям к оптимизируемым приборам, по графикам потребления всех приборов (для оценки суммарной потребляемой мощности) и пр. будут вводиться в программу, тем больший выигрыш будет у пользователя от оптимизации; с другой стороны, с увеличением объема данных резко сокращается количество пользователей, готовых выполнить работу по их вводу в программу. Более того, этот вопрос можно поставить так: насколько легко пользователь может определить значение того или иного входного параметра программы оптимизации? Так, величину потребляемой мощности стиральной машины определить несложно по техническому паспорту, а для определения коэффициента теплового сопротивления стенки холодильника пользователь должен обладать специальными знаниями и навы- ками. В то же время значения этих параметров (как будет показано ниже) необходимы для оптимизации работы холодильника.

Исходя из сказанного, можно сформулировать следующее утверждение: для практического применения методики оптимизации энергопотребления, необходимо минимизировать объем вводимых пользователем входных данных и, одновременно, предоставить пользователю доступные инструкции для определения значений входных параметров.

Рассмотрим это утверждение на примере цитируемой многими авторами работы [2]. При формализации задачи оптимизации в этой работе для каждого прибора вводится матрица, строки которой определяют все допустимые графики работы прибора. Эта матрица считается заданной и работа по составлению этой матрицы возлагается на пользователя. Если обычный пользователь начнет вводить данные в программу оптимизации по методике, предлагаемой в [2], то в процессе ввода неизбежно возникнут следующие трудности:

a) Допустим, что нужно заполнить указанную матрицу для стиральной машины так, чтобы стирка закончилась между десятью часами вечера и полуночью. При 10-ти минутном интервале оптимизации пользователю придется заполнить нулями и единицами 12 строк.

Ясно, что объем вводимых данных избыточен и в программе оптимизации должен присутствовать модуль, автоматически переводящий описание функциональных требований в матричное представление.

б) Рассмотрим ввод данных для оптимизации работы кондиционера таким образом, чтобы температура в комнате находилась в диапазоне 22-25 градусов. В цитируемой работе не предложено методики определения допустимости слу- 
чайно выбранного графика к условиям задачи.

В этом примере при избыточности объема вводимых данных не представлена методика определения самих данных, т.е. обычный пользователь не сможет оптимизировать работу своего кондиционера, пользуясь алгоритмом, предложенном в работе [2].

Следующий пример показывает, что в некоторых ситуациях матрицу [2] невозможно заполнить: одна и та же строка матрицы может быть, как допустимой, так и нет, в зависимости от условий, никак не связанных с работой прибора.

в) Рассмотрим следующую ситуацию: необходимо оптимизировать работу стиральной машины и электрической сушилки для одежды, так, чтобы операции стирки и последующей сушки закончились не позднее 23.00. Тогда строка в матрице, описывающей сушилку, работа которой начинается, например, в 21.00 будет допустимой, если операция стирки закончится к этому моменту и недопустимой в обратном случае.

Из рассмотренных примеров можно сделать вывод, что методику оптимизации энергопотребления, которую удобно применять на практике, нужно строить в следующем порядке:

1. Провести группировку приборов по способу задания функциональных требований;

2. Выяснить приемлемый для пользователя состав и вид входных данных;

3. Определить минимальный набор входных данных для формализации ограничения суммарной потребляемой мощности;

4. Сконструировать алгоритмы оптимизации, работающие с указанными выше входными данными.

Это означает, что первичным является вопрос о данных (по оптимизируемым приборам и ограничению на суммарную потребляемую мощность), которые может предоставить пользователь, а алгоритмы опными. Рассмотрим подробно каждый из перечисленных пунктов 1-4.

\section{2 Формализация функциональных требований к приборам}

Для формализации сгруппируем по способу задания функциональных требований, множество различных видов приборов в подмножества и определим для каждого из них структуру входных данных для программы оптимизации. При этом разделим входные данные на две части, описывающие паспортные характеристики прибора и функциональные требования пользователя к прибору.

\section{Приборы, не подлежащие оптимизации}

К этому подмножеству относятся приборы, для которых невозможно формализовать функциональные требования, либо, наоборот, приборы, для которых программа работы точно задана. В него включены все приборы, запуск и остановку которых, осуществляет пользователь (электрическая плита, компьютер, пылесос) и приборы с заданной автоматической программой работы, например, записывающий по таймеру магнитофон. К этому подмножеству также можно отнести и осветительные приборы [17].

\section{Приборы с ииклическим режимом работы}

К этому подмножеству относятся приборы, у которых цикл работы состоит из выполнения заданного (по потребляемой тимизации являются вторич-

энергии и времени выполнения) ряда операций: стиральные и посудомоечные машины, зарядные устройства и др.

Необходимый набор паспортных характеристик прибора для программы оптимизации, у этого подмножества можно представить следующим образом:

- время выполнения цикла работы (или каждой операции цикла);

- количество потребляемой энергии за цикл (или каждой операции цикла);

Например, цикл работы посудомоечной машины состоит из трех операций: нагрев воды, мытье посуды и сушка.

Функциональные требования к приборам этого подмножества определяются интервалом времени в течении которого прибор должен начать или закончить свою работу. Например, требуется зарядить ноутбук к 7-ми часам утра.

\section{Приборы с термостатическим управлением}

К этому виду относятся приборы, которые предназначены для поддержания температуры в заданных пределах, такие как кондиционеры, обогреватели, холодильники, морозильники. Можно определить алгоритм их включения и выключения, зависящий от температуры прибора (назовем ее «внутренняя температура») и температуры окружающей среды («внешняя температура»).

Паспортные данные этого подмножества приборов включают только потребляемую мощность, а функциональные требования заключаются в том,

Таблица

Внутренние и внешние температуры

\begin{tabular}{|c|c|c|c|}
\hline & Холодильник & Морозильник & $\begin{array}{c}\text { Кондиционер/ } \\
\text { нагреватель }\end{array}$ \\
\hline $\begin{array}{c}\text { Внутренняя } \\
\text { температура }\end{array}$ & $\begin{array}{c}\text { Температура } \\
\text { в камере }\end{array}$ & $\begin{array}{c}\text { Температура } \\
\text { в камере }\end{array}$ & $\begin{array}{c}\text { Температура } \\
\text { в комнате }\end{array}$ \\
\hline $\begin{array}{c}\text { Внешняя } \\
\text { температура }\end{array}$ & $\begin{array}{c}\text { Температура } \\
\text { в комнате }\end{array}$ & $\begin{array}{c}\text { Температура } \\
\text { в комнате }\end{array}$ & $\begin{array}{c}\text { Температура } \\
\text { на улице }\end{array}$ \\
\hline$\Theta_{\text {comf }}^{-}$ & 0 & -25 & 22 \\
\hline$\Theta_{\text {comf }}^{+}$ & 6 & -18 & 26 \\
\hline
\end{tabular}


что внутренняя температура прибора должна находиться в заданном диапазоне. В таблице конкретизируются понятия внутренней и внешней температуры и диапазона комфортных температур для основных приборов этого подмножества.

K перечисленным параметрам необходимо добавить еще два, характеризующих скорость изменения внутренней температуры, например, для холодильника - скорость повышения температуры, когда он не работает и понижения, когда работает. Об этих параметрах речь пойдет ниже, в разделе, посвященном созданным математическим моделям.

\section{Приборы с циклическим \\ режимом работы с возможностью прерывания и возобновления иикла}

K этому подмножеству можно отнести зарядные устройства (например, уже рассматриваемый процесс зарядки ноутбука в течении ночи можно прерывать и возобновлять), принтер (в тех случаях, когда имеется сервер печати и необходимо, например, распечатать определенное число страниц в ночное время) и др. В это подмножество так же можно включить и несколько «зависимых» приборов, т.е. приборов, у которых время начала работы одного прибора зависит от времени окончания работы другого.

\section{Приборы с несколькими режимами работы}

Это приборы, обладающие возможностью выполнить одну работу в разные промежутки времени с различным энергопотреблением. К этому подмножеству можно отнести как термостатические, так и приборы с циклическим режимом, например: кондиционер и обогреватель, зарядные устройства и другие приборы, имеющие несколько режимов.

В этом случае функциональные требования не изменятся, а к паспортным данным нужно добавить уже перечисленные данные для каждого режима.

\section{3 Формализация ограничения суммарной потребляемой мощности}

Учитывая принятое допущение о том, что в течение любого интервала оптимизации общая потребляемая мощность не меняется, можно перейти к эквивалентному ограничению на общую потребляемую энергию в течение интервала оптимизации. Тогда, при известном максимальном потреблении, эта задача сводится к задаче определения функции $A^{-}(t)$ - суммарного потребления в течении $t$-го интервала оптимизации всех приборов, не входящих в список оптимизируемых приборов. При этом нужно учитывать сформулированное утверждение о минимизации объема входных данных, вводимых пользователем.

Методам определения функции $A^{-}(t)$ посвящено немалое количество работ. В канонической, активно цитируемой и используемой работе [18] благодаря полной и детальной статистике по приборам и социальному составу жителей исследуемых домохозяйств построена математическая модель (модель «Капассо»), которая с высокой точностью прогнозирует графики потребления электроэнергии как суммарной, так и по отдельным группам приборов. Для РФ подобная статистика отсутствует, поэтому в данной работе предлагается использовать оценку функции $A^{-}(t)$ на основе методики расчета суточного профиля нагрузки домохозяйств [19]. Методика построена таким образом, чтобы минимизировать общее количество измерений. Однако, в результате обработки данных получается усредненный суточный профиль нагрузки, а для ограничения по суммарной потребляемой мощности нужна оценка максимального суточного профиля. Поэтому, потребовалась небольшая модифика- ция методики, заключающаяся в следующем: было сделано предположение, что значения потребляемой мощности, полученные при измерении, можно рассматривать как случайную величину, имеющую нормальное распределение. Тогда можно применить правило трех сигм и утверждать, что почти всегда максимальная потребляемая мощность будет меньше суммы средней мощности и утроенного среднеквадратичного отклонения. И, с помощью этого утверждения, была проведена оценка функции $A^{-}$ (t).

Таким образом, определены все структуры входных данных для программы оптимизации. С одной стороны, каждый из перечисленных параметров является необходимым, без них оптимизацию провести не возможно, а с другой - пользователь без труда сможет определить значение каждого параметра, т.к. возможно всего 4 варианта:

- значение параметра определяется предпочтениями пользователя (срок окончания программы стирки, диапазон комфортных температур для кондиционера);

- значение параметра можно найти в паспорте прибора (потребляемая мощность, время работы операции);

- параметры, относящиеся к ограничению на максимально потребление, определяются не пользователем, а соответствующими аудиторами, причем, аудит проводится только у нескольких представителей домохозяйств, в среднем составляющей единицы и иногда даже доли процентов;

- значения двух параметров для приборов с термостатическим управлением; ниже будет приведена простая (для пользователя) методика их определения.

Перейдем к рассмотрению созданной математической модели и алгоритмам оптимизации энергопотребления. 


\section{4 Алгоритм оптимизации} энергопотребления

Прежде чем рассматривать сами алгоритмы, необходимо сделать важное замечание, касающихся целевой (оптимизируемой) функции и общему виду целевой функции и неравенств.

Даже для небольшого домохозяйства и длительности оптимизации в несколько суток, общее количество ограничений составляет порядка $10^{2}-10^{4}$. Соответственно, необходимо учитывать проблему вычислительной сложности. Как отмечалось выше, задача оптимизации энергопотребления сводится к одной из стандартных оптимизационных задач, анализ которых показал, что наиболее эффективные и быстродействующие алгоритмы для такого количества ограничений имеются для задачи линейного программирования [20, 21]. Поэтому, алгоритм оптимизации проектировался так, чтобы полученные неравенства удовлетворяли условиям задачи линейного целочисленного программирования.

Алгоритм конструировался достаточно стандартным образом: по каждому из рассмотренных подмножеств видов приборов определялся их вклад в целевую функцию и ограничение по суммарной потребляемой мощности, а также составлялись неравенства, описывающие ограничения, вводимые на переменные и функциональные требования к приборам. Для этого введены переменные, описывающие состояние прибора (вкл/выкл) в течении каждого интервала оптимизации и с их помощью получены все перечисленные выражения (целевой функции как суммарной стоимости потребленной электроэнергии, и т.д.).

Рассмотрим особенности в конструировании алгоритма.

\section{Приборы с ииклическим} режимом работы

Основная трудность моделирования этой группы при- боров состояла в преобразовании полученных выражений к виду задачи линейного программирования. В результате появилась методика, идею которой рассмотрим на простом примере: необходимо оптимизировать работу прибора, цикл которого состоит из трех операций (каждая из которых занимает один интервал оптимизации) в течении 6-ти интервалов оптимизации.

Обозначим $a_{1}, a_{2}, a_{3}$ - потребление прибора на каждом интервале цикла, а $c_{1}, c_{2}, c_{3}$, $c_{4}, c_{5}, c_{6}-$ стоимости единицы электроэнергии на каждом интервале оптимизации. Введем четыре оптимизируемых переменных $e_{1}, e_{2}, e_{3}, e_{4} \in\{0 . .1\}$, соответствующих номеру интервала начала работы прибора, следующим способом: если прибор начинает работать с $l$-го интервала, то $e_{k}=1 ; k=l$ и $e_{k}=0 ; k \neq l$. Тогда общие ограничения на переменные $e_{i}$ можно записать так:

$$
\left\{\begin{array}{l}
0 \leq e_{1} \leq 1 \\
\cdots \\
0 \leq e_{4} \leq 1 \\
e_{1}+e_{2}+e_{3}+e_{4}=1 .
\end{array}\right.
$$

Выражение части целевой функции:

$$
\begin{aligned}
& e_{1} \cdot\left(c_{1} \cdot a_{1}+c_{2} \cdot a_{2}+c_{3} \cdot a_{3}\right)+ \\
& e_{2} \cdot\left(c_{2} \cdot a_{1}+c_{3} \cdot a_{2}+c_{4} \cdot a_{3}\right)+ \\
& e_{3} \cdot\left(c_{3} \cdot a_{1}+c_{4} \cdot a_{2}+c_{5} \cdot a_{3}\right)+ \\
& e_{4} \cdot\left(c_{4} \cdot a_{1}+c_{5} \cdot a_{2}+c_{6} \cdot a_{3}\right) \rightarrow \min .
\end{aligned}
$$

Выражения по вкладу в общее потребление на $k$-м интервале оптимизации $R(k)$ :

$$
\left\{\begin{array}{l}
R(1)=e_{1} \cdot a_{1} \\
R(2)=e_{1} \cdot a_{2}+e_{2} \cdot a_{1} \\
R(3)=e_{1} \cdot a_{3}+e_{2} \cdot a_{2}+e_{3} \cdot a_{1} \\
R(4)=e_{2} \cdot a_{3}+e_{3} \cdot a_{2}+e_{4} \cdot a_{1} \\
R(5)=e_{3} \cdot a_{3}+e_{4} \cdot a_{2} \\
R(6)=e_{4} \cdot a_{3} .
\end{array}\right.
$$

Таким образом, все полученные выражения линейны, относительно введенных переменных и удовлетворяют условиям задачи линейного программирования.

\section{Приборы с термостатическим управлением}

Главная трудность, возникающая при моделировании приборов из этого подмножества, заключается в математическом описании функционального ограничения на внутреннюю температуру: она должна находиться в заданном диапазоне. Решение этой задачи отталкивалось от упрощенной математической модели процесса поддержания внутренней температуры прибора, описываемого дифференциальным уравнением 1-го порядка, которая апробирована на реальных данных и широко используется в исследованиях по энергопотреблению [22].

В результате было показано, что достаточно проверить выполнение неравенства относительно внутренней температуры на концах всех интервалов оптимизации и получена зависимость внутренней температуры от графика использования прибора. Это выражение является линейным и удовлетворяет условиям задачи линейного программирования. Также оно содержит два комплексных параметра, описывающих теплоемкость, тепловое сопротивление и коэффициент полезного действия прибора. Для определения значений этих параметров предложена простая методика: пользователю необходимо провести 3 измерения внутренней температуры прибора с интервалом, равным интервалу оптимизации при включенном и выключенном приборе. На примере комнатного обогревателя и величины интервала оптимизации в 10 минут, пользователю нужно провести следующие действия:

- измерить температуру в комнате,

- включить обогреватель на 10 минут,

- измерить температуру в комнате,

- выключить обогреватель и измерить температуру в комнате через 10 минут. 
Этих измерений достаточно для определения необходимых параметров; с одной стороны это - необходимый минимум, с другой - измерения просты и доступны для пользователя.

Также были получены выражения для следующих важных ситуаций использования указанного подмножества приборов:

1. Случай работы прибора в заданных промежутках времени, например, в интервале с 8.00 до 19.00 температура в помещении должна находиться в рамках комфортного диапазона.

2. Случай работы нескольких приборов в общей окружающей среде, например, поддержание температуры в одной комнате несколькими обогревателями.

3. Ситуации, когда внутренняя температура одного прибора является внешней температурой для другого. Например, оптимизация совместной работы холодильника и кондиционера, которые расположены в одном помещении.

\section{Приборы с ииклическим режимом работы с возможностью прерывать и возобновлять цикл}

При моделировании прибор из этого подмножества представлялся в виде нескольких приборов, цикл работы которых нельзя прерывать. За исключением того, что полученные выражения оказались еще более громоздкими, чем у приборов с циклическим режимом работы, никаких трудностей не возникает.

\section{Приборы с несколькими режимами работы}

Приборы из этого подмножества моделировались аналогичным образом: каждый прибор представлялся в виде нескольких приборов (по количеству режимов работы) и вводилось дополнительное ограничение, запрещающее возможность работы более одного прибора для любого интервала оптимизации.
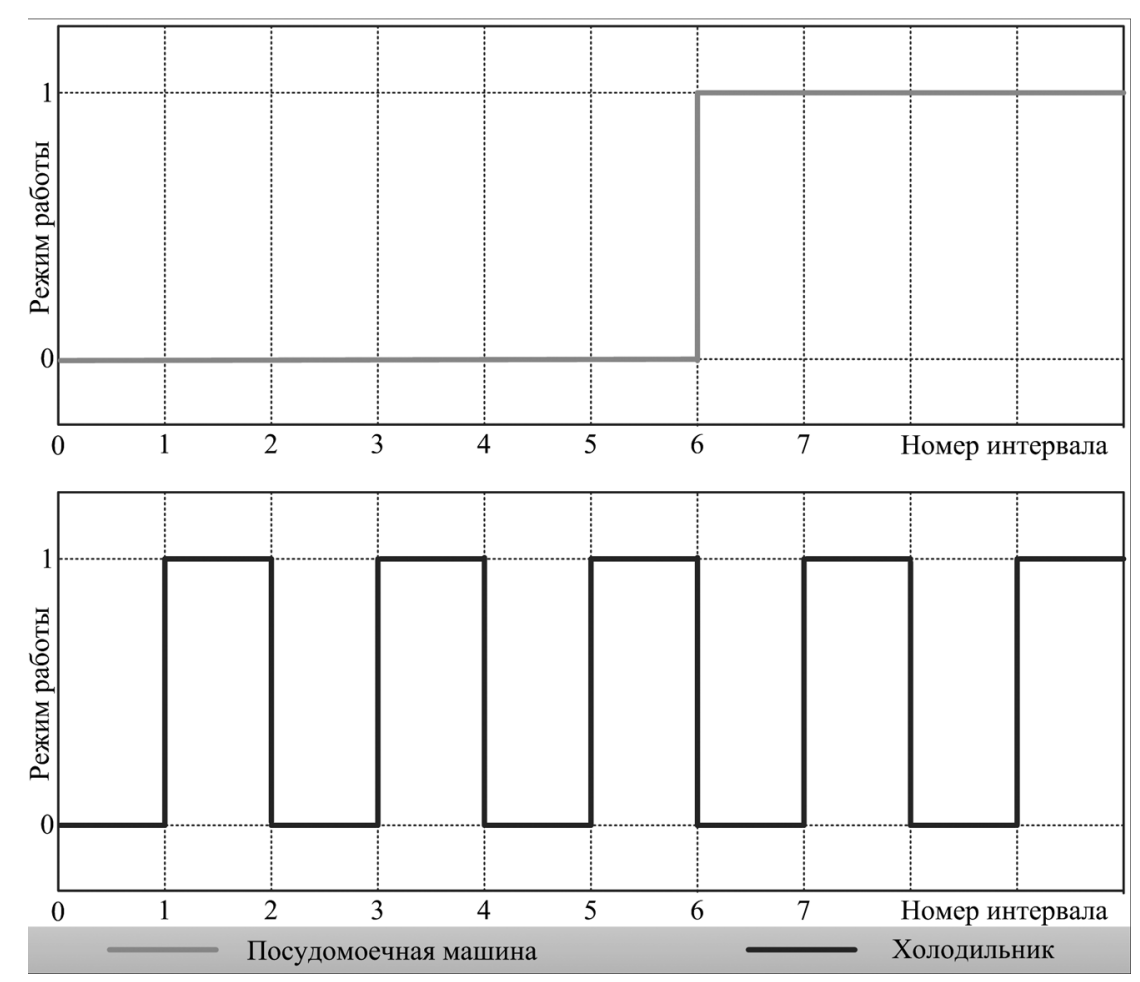

Рис. Графики зависимости работы приборов от номера интервала оптимизации

\section{2. Практический пример оптимизации энергопотребления}

Работоспособность полученного алгоритма была проверена на упрощенном примере оптимизации работы холодильника и посудомоечной машины (с двумя режимами работы) в течение пяти часов.

При 30-ти минутном интервале оптимизации были составлены выражения целевой функции и 76 неравенств, описывающих ограничения функциональные ограничения приборов и ограничение на максимально потребляемую мощность. Полученные выражения были объединены, переведены в матричный вид и с помощью программы Matlab найдено оптимальное решение поставленной задачи, точнее несколько оптимальных решений. На рисунке приведены графики работы приборов в одном из оптимальных вариантов.

Анализ полученного решения показал, что при умень- шении интервала оптимизации суммарная стоимость электроэнергии будет уменьшаться, поэтому при практическом применении значение величины интервала оптимизации нужно задавать меньше, чем в приведенном примере (около 1-5 минут), но так, чтобы решение задачи выполнялось за приемлемое время.

\section{Заключение}

1. При исследовании существующих методов оптимизации энергопотребления в домохозяйствах была выявлена проблема, заключающаяся в сложности применения этих методов на практике.

2. Последующий анализ позволил сформулировать основные принципы построения методики оптимизации, удобной для практического применения.

3. Для формализации функциональных требований проведено разделение множества различных видов приборов на подмножества, для каждого из 
которых определена структура входных данных.

4. Разработана методика прогнозирования графика максимальной суммарной мощности.
5. Разработаны методики оптимизации энергопотребления для каждого из выделенных подмножеств бытовых приборов.

6. Выполнено моделирование полученных алгорит- мов оптимизации, которое показало их работоспособность, эффективность и возможность их практического применения без какой-либо адаптации.

\section{Литература}

1. $\mathrm{Na} \mathrm{Li,} \mathrm{Lijun} \mathrm{Chen,} \mathrm{Steven} \mathrm{H.} \mathrm{Low} \mathrm{Optimal}$ Demand Response Based on Utility Maximization in Power Networks Power and Energy Society General Meeting// IEEE Power and Energy Society General Meeting. 2011. P. 1-8.

2. Volkova I.O., Gubko M.V., Salnikova E.A. Active consumer: optimization problems of power consumption and self-generation // Automation and remote control. 2014. 75. 3. P. 551-562. doi: http://dx.doi.org/10.1134/S0005117914030114.

3. Albani M.H., El-Saadany E.F. A summary of demand response in electricity markets// Electric power systems Research. 2008. 11. 78. P. 19891996.

4. Ann-Piette M., Ghatikar G., Kiliccote S., Watson D., Koch E., Hennage D. Design and operation of an open, interoperable automated demand response infrastructure for commercial buildings // J. Comput. Inf. Sci. Eng. Jun. 2009. Vol. 9. P. 1-9.

5. Saeid Bashash, Hosam K. Fathy Modeling and Control Insights into Demand-side Energy Management through Setpoint Control of Thermostatic Loads// American Control Conference on O'Farrell Street. San Francisco. CA. USA. 2011 June 29 - July 01. P. 4546-4553. doi: http://dx.doi. org/10.1109/ACC.2011.5990939.

6. Bradac Z., Kaczmarczyk V., Fiedler P. Optimal scheduling of domestic appliances via MILP// Energies. 2015. 8. 1. P. 217-232. doi: http://dx.doi. org/10.3390/en8010217.

7. S-C Chan Load/price forecasting and managing demand response for smart grids: Methodologies and challenges// Signal processing magazine. 2012. 29. 5. P. 68-85. doi: http://dx.doi.org/10.1109/ MSP.2012.2186531.

8. A.J. Conejo, J.M. Morales, L. Baringo Realtime demand response model// Smart grid, IEEE transactions. 2010. 1. 3. P. 236-242. doi: http:// dx.doi.org/10.1109/TSG.2010.2078843.

9. R.S. Ferreira, L.A.N. Barroso, M.M. Carvalho Demand response models with correlated price data: a robust optimization approach// App. Energy. 2012. 96. P. 133-149. doi: http://dx.doi.org/10.1016/j. apenergy.2012.01.016.

10. S. Gottwalt Demand side management - a simulation of household behavior under variable prices// Energy policy. 2011. 39. 12. P. 3-8174. doi: http://dx.doi.org/10.1016/j.enpol.2011.10.016.

11. B. Li Predicting user comfort level using machine learning for smart grid environments//

\section{References}

1. Na Li, Lijun Chen, Steven H. Low Optimal Demand Response Based on Utility Maximization in Power Networks Power and Energy Society General Meeting// IEEE Power and Energy Society General Meeting. 2011. P. 1-8.

2. Volkova I.O., Gubko M.V., Salnikova E.A. Active consumer: optimization problems of power consumption and self-generation. Automation and remote control. 2014. 75. 3. P. 551-562. doi: http://dx.doi.org/10.1134/S0005117914030114.

3. Albani M.H., El-Saadany E.F. A summary of demand response in electricity markets// Electric power systems Research. 2008. 11. 78. P. 19891996.

4. Ann-Piette M., Ghatikar G., Kiliccote S., Watson D., Koch E., Hennage D. Design and operation of an open, interoperable automated demand response infrastructure for commercial buildings.J. Comput. Inf. Sci. Eng. Jun. 2009. Vol. 9. P. 1-9.

5. Saeid Bashash, Hosam K. Fathy Modeling and Control Insights into Demand-side Energy Management through Setpoint Control of Thermostatic Loads// American Control Conference on O'Farrell Street. San Francisco. CA. USA. 2011 June 29 - July 01. P. 4546-4553. doi: http://dx.doi.org/10.1109/ACC.2011.5990939.

6. Bradac Z., Kaczmarczyk V., Fiedler P. Optimal scheduling of domestic appliances via MILP// Energies. 2015. 8. 1. P. 217-232. doi: http://dx.doi.org/10.3390/en8010217.

7. S-C Chan Load/price forecasting and managing demand response for smart grids: Methodologies and challenges// Signal processing magazine. 2012. 29. 5. P. 68-85. doi: http://dx.doi. org/10.1109/MSP.2012.2186531.

8. A.J. Conejo, J.M. Morales, L. Baringo Realtime demand response model// Smart grid, IEEE transactions. 2010. 1. 3. P. 236-242. doi: http:// dx.doi.org/10.1109/TSG.2010.2078843.

9. R.S. Ferreira, L.A.N. Barroso, M.M. Carvalho Demand response models with correlated price data: a robust optimization approach. App. Energy. 2012. 96. P. 133-149. doi: http://dx.doi. org/10.1016/j.apenergy. 2012.01.16.

10. S. Gottwalt Demand side management a simulation of household behavior under variable prices// Energy policy. 2011. 39. 12. P. 3-8174. doi: http://dx.doi.org/10.1016/j.enpol.2011.10.016.

11. B. Li Predicting user comfort level using machine learning for smart grid environments// 
Innovative smart grid technologies (ISGT 2011). P. 1-6.

12. J.M. Lujano-Rojas Optimum residential load management strategy for real time pricing demand response programs// Energy policy. 2012. 45. P. 671-679. doi: http://dx.doi.org/10.1016/j. enpol.2012.03.019.

13. Amir-Hamed Mohsenian-Rad, Alberto LeonGarcia Optimal Residential Load Control With Price Prediction in Real-Time Electricity Pricing Environments// IEEE Transactions on smart grid. Sept. 2010. Vol.1. No.2. P. 120-133. doi: http:// dx.doi.org/10.1109/TSG.2010.2055903.

14. M.A.A. Pedrasa, T.D. Spooner, I.F. MaxGill Scheduling of demand side resources using binary particke swarm optimization// IEEE Transactions on Power Systems. Aug. 2009. Vol. 24. No. 3. P. 1173-1181.

15. B. Ramanathan, V. Vittal A framework for evaluation of advanced direct load control with minimum disruption// IEEE Transactions on Power Systems. Nov. 2008 Vol. 23. No.4. P. 1681-1688. doi: http://dx.doi.org/10.1109/TPWRS.2008.2004732.

16. K.C. Sou Scheduling smart home appliances using mixed integer linear programming// 50th IEEE conference on decision and control and European control conference (CDC-ECC 2011). P. 5144-5149. doi: http://dx.doi.org/10.1109/ CDC.2011.6161081.

17. Scott Ziegenfus Demand Response And Light Control // ASHRAE Journal. November. 2012. P B20-B24.

18. Capasso A., Grattieri W., Lamedica R., Prudenzi A. A bottom-up approach to residential load modeling// IEEE Transactions on Power Systems. Sept. 1994. Vol. 2. P. 957-965. doi: http:// dx.doi.org/10.1109/59.317650.

19. Гребенюк Г.Г., Ковалев С.П., Крыгин А.А., Середа Л.А. Организация энергоменеджмента и планирование электрической нагрузки домохозяйств// Энергобезопасность и энергосбережение. 2015. № 3. С. 22-27.

20. Зоркальцев В.И., Филатов А.Ю. Новые варианты двойственных алгоритмов внутренних точек для систем линейных неравенств // Журнал вычислительной математики и математической физики. 2004. Том 44. № 7. С. 1234-1243.

21. Усков Е.И. Численное сравнение оптимизационных алгоритмов // Теоретические и прикладные задачи нелинейного анализа. ВЦ РАН Москва. 2012. С. 118-131.

22. Callaway D. S. Tapping the energy storage potential in electric loads to deliver load following and regulation, with application to wind energy // Energy Conversion and Management. 2009, Vol. 50, P. 1389-1400. doi: http://dx.doi.org/10.1016/j. enconman.2008.12.012
Innovative smart grid technologies (ISGT 2011) P. 1-6.

12. J.M. Lujano-Rojas Optimum residential load management strategy for real time pricing demand response programs// Energy policy. 2012. 45. P. 671-679. doi: http://dx.doi.org/10.1016/j. enpol.2012.03.019.

13. Amir-Hamed Mohsenian-Rad, Alberto LeonGarcia Optimal Residential Load Control With Price Prediction in Real-Time Electricity Pricing Environments// IEEE Transactions on smart grid. Sept. 2010. Vol. 1. No. 2. P. 120-133. doi: http:// dx.doi.org/10.1109/TSG.2010.2055903.

14. M.A.A. Pedrasa, T.D. Spooner, I.F. MaxGill Scheduling of demand side resources using binary particke swarm optimization// IEEE Transactions on Power Systems. Aug. 2009. Vol. 24. No. 3. P. 1173-1181.

15. B. Ramanathan, V. Vittal A framework for evaluation of advanced direct load control with minimum disruption// IEEE Transactions on Power Systems. Nov. 2008 Vol. 23. No.4. P. 1681-1688. doi: http://dx.doi.org/10.1109/TPWRS.2008.2004732.

16. K.C. Sou Scheduling smart home appliances using mixed integer linear programming// 50th IEEE conference on decision and control and European control conference (CDC-ECC 2011). P. 5144-5149. doi: http://dx.doi.org/10.1109/ CDC.2011.6161081.

17. Scott Ziegenfus Demand Response And Light Control. ASHRAE Journal. November. 2012. P. B20-B24.

18. Capasso A., Grattieri W., Lamedica R., Prudenzi A. A bottom-up approach to residential load modeling// IEEE Transactions on Power Systems. Sept. 1994. Vol. 2. P. 957-965. doi: http:// dx.doi.org/10.1109/59.317650.

19. Grebenyuk G.G., Kovalev S.P., Krygin A.A., Sereda L.A. Organizatsiya energomenedzhmenta i planirovaniye elektricheskoy nagruzki domokhozyaystv// Energobezopasnost' i energosberezheniye. 2015. No. 3. P. 22-27. (In Russ.)

20. Zorkal'tsev V.I., Filatov A.YU. Novyye varianty dvoystvennykh algoritmov vnutrennikh tochek dlya sistem lineynykh neravenstv. ZHurnal vychislitel'noy matematiki i matematicheskoy fiziki. 2004. Tom 44. No. 7. P. 1234-1243. (In Russ.)

21. Uskov E.I. CHislennoye sravneniye optimizatsionnykh algoritmov. Teoreticheskiye i prikladnyye zadachi nelineynogo analiza. VTS RAN Moskva. 2012. P. 118-131. (In Russ.)

22. Callaway D. S. Tapping the energy storage potential in electric loads to deliver load following and regulation, with application to wind energy. Energy Conversion and Management. 2009, Vol. 50, P. 1389-1400. doi: http://dx.doi.org/10.1016/j. enconman. 2008.12.012 
Сведения об авторе

Георгий Григорьевич Гребенюк

ИПУ им. В.А. Трапезникова РАН,

Москва, Россия

Эл.noчma: grebenuk@lab49.ru

Сергей Максимович Никишов

ИПУ им. В.А. Трапезникова РАН,

Москва, Россия

Эл.noчma:nikishov@lab49.ru

Андрей Александрович Крыгин

ИПУ им. В.А. Трапезникова РАН,

Москва, Россия

Эл. nочma: andreyakr@yandex.ru

Леонид Анатольевич Середа

ИПУ им. В.А. Трапезникова РАН,

Москва, Россия

Эл.noчma: sereda@lab49.ru
Information about the authors

Georgiy G. Grebenuk

$V$. A. Trapeznikov Institute of Control Sciences of RAS, Moscow, Russia

E-mail: grebenuk@lab49.ru

Sergey M. Nikishov

$V$. A. Trapeznikov Institute of Control Sciences of

RAS, Moscow, Russia

E-mail:nikishov@lab49.ru

Andrey A. Krygin

$V$. A. Trapeznikov Institute of Control Sciences of RAS, Moscow, Russia

E-mail: andreyakr@yandex.ru

Leonid A. Sereda

$V$. A. Trapeznikov Institute of Control Sciences of

RAS, Moscow, Russia

E-mail: sereda@lab49.ru 